\title{
Single Use (Disposable) Duodenoscope: Recent Development and Future
}

\author{
Kihyun Ryu' and Sunguk Jang ${ }^{2}$ \\ ${ }^{1}$ Department of Gastroenterology, Konyang University College of Medicine, Daejeon, Korea, ${ }^{2}$ Digestive Disease Institute, Cleveland \\ Clinic, Cleveland, OH, USA
}

Unlike simple forward-viewing endoscopes such as gastroscope or colonoscope, duodenoscope houses much more complex design to fulfil its function. This design differences leave duodenoscopes more prone to contamination from inadequate disinfection process and potential dissemination of pathogens. Recent reports on dissemination of infection through the duodenoscope mandated an overhaul of duodenoscope utilization including development of a disposable duodenoscope. This article reviews the current state of disposable duodenoscope development, including reported early efficacy as well as its future direction and utilization. Clin Endosc 2022;55:191-196

Key Words: Disposable equipment; Duodenoscopes; Infectious disease transmission

\section{INTRODUCTION}

Gastrointestinal endoscopy involves the process of inserting an endoscope via patients' oropharyngeal space where microbes reside, prompting the concern of spreading various infections. Due to the significant risk of transmitting pathogens between the individuals undergoing endoscopic procedure, high level disinfection (HLD) process of endoscopes is of utmost importance. To that end, careful endoscopy reprocessing protocols have been established to mitigate this adverse outcome. The bulk of these protocols however, focus on the disinfection process of forward-viewing endoscope such as gastroscope and colonoscope.

The duodenoscope is a side-viewing endoscope, where its

Received: February 9, 2021 Revised: March 14, 2021

Accepted: March 15, 2021

Correspondence: Sunguk Jang

Digestive Disease Institute, Cleveland Clinic, 9500 Euclid Avenue A-31, Cleveland $\mathrm{OH}, 44195$ USA

Tel: +1-216-407-3755, Fax: +1-216-444-2243, E-mail: jangs@ccf.org

ORCID: https://orcid.org/0000-0001-9837-0322

It is the invited review article.

(c) This is an Open Access article distributed under the terms of the Creative Commons Attribution Non-Commercial License (http://creativecommons.org/ licenses/by-nc/3.0) which permits unrestricted non-commercial use, distribution, and reproduction in any medium, provided the original work is properly cited. camera and working channel exit point are located at the side of scope tip, rather than at its end. In addition, the presence of "elevator" to adjust the angle of accessory device approach further adds to the complexity of its construct, as well as its cleansing process. There are potential "blind spots" where collection of debris and bacterial flora can gather and serve as a source of microbial transmission. Thus, standard cleaning and disinfection methods may be insufficient for a duodenoscope. Due to its mechanical complexity and sensitive component, high-temperature sterilization is not feasible. ${ }^{1,2}$ In recent years, carbapenem resistant Enterobacteriaceae (CRE) infection outbreak among the patients who underwent endoscopic retrograde cholangiopancreatography (ERCP) have been reported, further substantiating the concern. ${ }^{3-6}$ Furthermore, another study linked the death of dozens to multidrug-resistant organisms after ERCP. In this review, we discuss currently available technologies and innovative endeavors with duodenoscope design to mitigate this potentially life-threatening adverse outcome.

\section{THE MAGNITUDE OF THE PROBLEM}

A Dutch medical group first attributed the transmission of infection in patients who underwent ERCP to the newly 
distributed, fixed-cap style duodenoscope (TJF-Q180V; Olympus Corporation, Tokyo, Japan ) in 2010. ${ }^{8}$ The transmission of Verona Integron-encoded Metallo- $\beta$-lactamase (VIM)-2-producing Pseudomonas aeruginosa was attributed to the unresolved bioburden within duodenoscope. Subsequently, the Dutch Ministry of Health conceded that endoscopic procedures do not have sufficient evidence to prove the validity and safety of current reprocessing instructions. ${ }^{9}$ Since then, a group of endoscopy manufacturers have issued two warnings to the European Endoscopy Center in 2013 and 2014. The warnings however were limited to the latest generation of duodenoscope at that time and potential inadequacy of its reprocessing process in infection prevention. In 2012, a series of US-based infections were reported in Massachusetts, New York, and Pennsylvania, raising similar concerns to the Dutch counterparts. In addition, most of the US-based cases were characterized by a unique antibiotic resistance pattern, based on in-vitro examination of the pathogens. ${ }^{10}$ In the end, these findings forced three side-viewing duodenoscope manufacturers to concede a real concern with the duodenoscope as a vector of concomitant infections with contamination. ${ }^{11}$

In 2018, 73 Dutch centers reported their findings of a nationwide cross-sectional study on the prevalence of bacterial contamination of reprocessed duodenoscopes. ${ }^{11}$ The samplings were performed from duodenoscopes that had undergone reprocessing such as high level disinfection or drying in the storage cabinet. At least two duodenoscopes were chosen for each center, including the newest Olympus TJFQ180V if available in their endoscopic procedure sites. They evaluated AM20 (microbial growth with $\geq 20 \mathrm{CFU} / 20 \mathrm{~mL}$ of any type of microorganism; ESGE guideline and Dutch De Stuurgroep Flexibele Endoscopen Reiniging en Desinfectie [SFERD] handbook) and microbes of gastrointestinal origin (MGO) (presence of microbial growth ( $\geq 1 \mathrm{CFU} / 20$ $\mathrm{mL}$ ) of gastrointestinal and/or oral microorganisms). Their findings showed AM20 contamination in 39\% of patient-ready duodenoscope, and 15\% of reprocessed/cleaned duodenoscopes harbored MGO, indicating the presence of organic residue of previously treated patients. In terms of prevalence, MGO detection was noted from cleansing brush (12\%), protection cap (11\%), air/water channel (5\%), biopsy channel (3\%), and suction channel (3\%). The detection rate of MGO was highest in brush (10\%), followed by biopsy protection cap (5\%), forceps elevator (5\%), biopsy channel (4\%), suction channel (4\%). Douglas et al. studied the effect of double-reprocessing high-level disinfection on a duodenoscope contamination. ${ }^{12}$ They reported the contamination rates between 4.8-9.4\% through the 3 phase study, which was lower than the rate reported by Ross et al. which was
$13.1 \% .^{10}$ Several studies identified the presence of biofilm, in which bacterial pathogens can survive despite standard disinfection process, as the main culprit of these worrisome findings. ${ }^{8,13,14}$

\section{PATHOGENS AND CLINICAL IMPLICATION}

Among the infectious bacteria, CRE, also known as "superbug", has recently garnered much concern. It was first reported in 2013 when the exposure to duodenoscope was identified as the culprit of transmission. ${ }^{4}$ In this outbreak of 39 cases, 35 patients were exposed to duodenoscope in one hospital. In addition, New Delhi metallo- $\beta$-lactamase (NDM)-producing Escherichia coli also was identified from a reprocessed duodenoscope. The clinical implication of CRE is significant in that its resistance to carbapenem, a broad-spectrum antibiotic with a robust treatment spectrum, renders medical community with limited treatment options. In Korea for example, CRE infection is designated as 'legal communicable disease' since 2010, and its outcomes are closely monitored with a designated surveillance system. In 2017, CRE was designated to infectious disease, Class III, requiring a mandatory surveillance system. As of 2020, CRE infection was elevated to Class II infectious disease in Korea. Specifically, Carbapenemase producing carbapenem-resistant Enterobacteriaceae is a type of CRE that produces enzymes to degrade a wide array of antibiotics, and also has the ability to transmit its resistance profile to other pathogens.

HLD defect rates are used as surrogate markers of scope related infection. One center recorded an HLD deficiency rate of $1.9 \%$ per year in 1500 consecutive cultures. ${ }^{10}$ Another center that performed ethylene oxide sterilization after the outbreak of CRE infection showed positive culture rate in the $1.2 \%$ range over 18 months form 84 duodenoscopes in their monthly culture analysis. ${ }^{15}$ During ERCP, CRE transmission after its use within contaminated duodenum(confirmed retrospectively) was $14.4 \%$ during biliary stenting. The inpatient status was identified as an independent risk factor (odds ratio 3.74, 95\% confidence interval 1.15-12.12) for CRE infection. The diagnosis of cholangiocarcinoma was also associated with an increased risk CRE infection. ${ }^{16}$

\section{DISPOSABLE SINGLE USE DUODENOSCOPE}

The argument for a single use duodenoscope is based on the validated findings of unsatisfactory level of disinfection of 
reprocessed duodenoscopes with currently available cleansing methods. At present, two broader options of disposable duodenoscope exist: Use of disposable pieces in currently available duodenoscopes, and the utilization of a single use duodenoscope that can be discarded after one-time use.

In April 2019, the US Food and Drug Administration (FDA) recommended that hospitals and endoscopy facilities should transition to innovative duodenoscope designs that include disposable components such as disposable endcaps or to fully disposable duodenoscopes when they become available. Although it is not a part of gastrointestinal endoscopy, a fiberoptic endoscope has also been developed and used in other specialties. These are single-use bronchoscope (aScope; Ambu A/S, Ballerup, Denmark) and single-use digital flexible ureteroscope (LithoVue; Boston Scientific, Marlborough, MA, USA). Both disposable endoscopes reported comparable performance and clinical effects with conventional fiberoptic endoscopes. ${ }^{17,18}$ In addition, there are reports that they have the effect of reducing overall medical costs in certain circumstances. ${ }^{19,20}$ In December 2019, FDA cleared first fully disposable duodenoscope, eliminating the potential for transmission of pathogens from ineffective reprocessing. To date, the FDA has cleared six duodenoscopes with disposable components that facilitate reprocessing (Table 1).

Muthusamy et al. performed a multi-center clinical evaluation on a single-use duodenoscope for ERCP in 6 academic medical centers with 7 expert endoscopists in US. ${ }^{21}$ They used the EXALT Model D single-use duodenoscope (Boston Scientific, Marlborough, MA, USA). Of 60 cases of ERCP with the EXALT model, 58 (96.7\%) cases were successfully completed with the use of the single use duodenoscope, while 2 cases (3.3\%) required transition to the traditional reusable duodenoscope after the attempts with single-use duodenoscope were not successful. The first case of crossover occurred during dilation of high-grade biliary stricture of a peripheral left-sided intrahepatic bile duct with primary sclerosing cholangitis history and reported to be associated with the duodenoscope being hard to torque and maneuver, possibly due to shaft stiffness. In the second crossover case, initial cannulation with both single-use duodenoscope and reusable duodenoscope (TJFQ180V; Olympus Corporation, Tokyo, Japan) were unsuccessful through the native papilla. The distribution of procedural complexity - based on American Society for Gastrointestinal Endoscopy guideline - was as followed: 7 patients (11.7\%) with complexity grade 1, 26 patients (43.3\%) with grade 2, 26 patients (43.3\%) with grade 3 , and a patient (1.7\%) with grade 4 (most complex). Two crossover cases were grade 2 and grade 3. ${ }^{22}$ Median overall satisfaction with the disposable duodenoscope was 9 when evaluated on Likert scale of 1 (unsatisfied) to 10 (very satisfied).
Ross et al. ${ }^{23}$ performed a comparative study between a single use duodenoscope and 3 models of reusable duodenoscopes for ERCP; EXALT Model D, TJF-Q180V reusable duodenoscope, ED-3470TK reusable duodenoscope (Pentax Corporation, Tokyo, Japan), and ED-530XT reusable duodenoscope (Fujifilm Holdings Corporation, Tokyo, Japan). They conducted a comparative bench stimulation study by 6 expert endoscopists. They rated various aspects of these scopes including navigational ease, mechanical control, and image quality on a scale of 1 (worst) to 10 (best). The overall performance ratings by tasks are similar (median, 8.0-10.0). Navigation/maneuverability ratings were lower for the disposable duodenoscope than for the three reusable duodenoscopes (median, 8.0, 10.0, 9.0 , and 9.0, respectively; $p<0.01$ ). Ratings tip control was similar for all duodenoscopes (median, 9.0-10.0; $p=0.77$ ). Image quality for 1 reusable duodenoscope (ED-3470TK) was rated lower than that of disposable and reusable duodenoscope one of the other two (median, respectively, 8.0, 9.0, 9.0 and 9.0; $p<0.01$ ). Thus, the median image quality score of single use duodenoscope was one point lower (score of 4 ) than the score expected with reusable duodenoscope (the score of 5). Similarly, the median score on the "elevator function" of single use duodenoscope was rated as 4 out of 5 . The clinical significance however of these scores, is not clear.

\section{CURRENT BARRIERS AND FUTURE}

The cost remains as one the biggest obstacles in the dissemination of single use duodenoscope. Bang et al. reported that each centers have different opinions for the cost of disposable duodenoscopes, as the cost per procedure depends on the number of cases performed with each scope. ${ }^{24}$ Using activity-based costs and financial models, the cost per case for the institutions performing at the $25^{\text {th }}$ percentile of ERCP procedural volume in US (less than 50 cases per year), the cost per procedure ranged from $\$ 1,318$ to $\$ 2,068$. For the institutions operating at the $75^{\text {th }}$ percentile of procedural volume (between 125 and 150 cases per year), the price was ranged from $\$ 797$ to $\$ 1,547$, based on the infection rates of $0.4 \%$ to $1 \%$ from published literature. ${ }^{11,16,25}$ The study set the lifecycle (longevity) of duodenoscope at 3 years and with cost of an individual duodenoscope at $\$ 35,000$ ( $\$ 11,667 /$ year), and annual maintenance and repair costs of $\$ 1,451$ per scope. To obtain the overall cost of reusable duodenoscope, they added annual scope washer maintenance costs $(\$ 8,000)$, cleaning supplies $(\$ 10,424)$, labor costs $(\$ 60,230)$. To account for cost attributable to adverse outcome of procedure related infection, the study group also added the treatment of cholangitis costs $(\$ 125,000$ per case, which included 2-day intensive care unit stay). Assuming that 
Table 1. FDA Cleared Duodenoscopes with Disposable Components

\begin{tabular}{|c|c|c|c|c|c|c|}
\hline Trade Name & $\begin{array}{c}\text { Duodenoscope } \\
\text { model aScope } \\
\text { Duodeno }\end{array}$ & $\begin{array}{l}\text { EXALT Model } \\
\text { D Single-Use } \\
\text { Duodenoscope }\end{array}$ & $\begin{array}{c}\text { Duodenoscope } \\
\text { model ED-580XT }\end{array}$ & $\begin{array}{c}\text { Evis Exera III Du- } \\
\text { odenovideoscope } \\
\text { Olympus TJF- } \\
\text { Q190V }\end{array}$ & $\begin{array}{c}\text { Duodenoscope } \\
\text { model ED34-i10T }\end{array}$ & $\begin{array}{c}\text { Duodenoscope } \\
\text { model ED34- } \\
\text { i10T2 }\end{array}$ \\
\hline Manufacturer & $\begin{array}{c}\text { Ambu Innovation } \\
\mathrm{GmbH}\end{array}$ & $\begin{array}{c}\text { Boston Scientific } \\
\text { Corporation }\end{array}$ & $\begin{array}{c}\text { Fujifilm } \\
\text { Corporation }\end{array}$ & $\begin{array}{l}\text { Olympus Medical } \\
\text { Systems }\end{array}$ & Pentax Medical & Pentax Medical \\
\hline $\begin{array}{l}\text { Disposable } \\
\text { components }\end{array}$ & Fully disposable & Fully disposable & Disposable endcap & Disposable endcap & Disposable endcap & $\begin{array}{l}\text { Disposable } \\
\text { elevator }\end{array}$ \\
\hline $\begin{array}{l}\text { Retro viewing } \\
\text { angle }\left(^{\circ}\right)\end{array}$ & 6 & 5 & 5 & 15 & 10 & 10 \\
\hline Field of view $\left(^{\circ}\right)$ & 130 & 108 & 100 & 100 & 100 & 100 \\
\hline $\begin{array}{l}\text { Depth of field } \\
(\mathrm{mm})\end{array}$ & & $5-60$ & $4-60$ & $5-60$ & $4-60$ & $4-60$ \\
\hline $\begin{array}{r}\text { Distal end outer } \\
\text { diameter }(\mathrm{mm})\end{array}$ & 13.7 & 15.1 & 13.1 & 13.5 & 13.6 & 13.6 \\
\hline $\begin{array}{l}\text { Insertion tube } \\
\text { outer diameter } \\
(\mathrm{mm})\end{array}$ & 11.3 & 11.3 & 11.3 & 11.3 & 11.6 & 11.6 \\
\hline $\begin{array}{l}\text { Working length } \\
(\mathrm{mm})\end{array}$ & 1240 & 1240 & 1250 & 1240 & 1250 & 1250 \\
\hline $\begin{array}{l}\text { Working } \\
\text { channel inner } \\
\text { diameter }(\mathrm{mm})\end{array}$ & 4.2 & 4.2 & 4.2 & 4.2 & 4.2 & 4.2 \\
\hline $\begin{array}{l}\text { Angulation } \\
\text { range } U p\left(^{\circ}\right)\end{array}$ & 120 & 120 & 120 & 120 & 120 & 120 \\
\hline $\begin{array}{l}\text { Angulation } \\
\text { range Down }\left({ }^{\circ}\right)\end{array}$ & 90 & 90 & 90 & 90 & 90 & 90 \\
\hline $\begin{array}{l}\text { Angulation } \\
\text { range Right }\left(^{\circ}\right)\end{array}$ & 110 & 110 & 110 & 110 & 105 & 105 \\
\hline $\begin{array}{l}\text { Angulation } \\
\text { range Left }\left(^{\circ}\right)\end{array}$ & 90 & 90 & 90 & 90 & 90 & 90 \\
\hline
\end{tabular}

each reusable duodenoscope is used approximately 200 times per year, the substitution with single use duodenoscope would incur a cost of $\$ 367,200$ (\$612 per procedure) over 3-year period. It is important to note that their calculation is based on high-volume endoscopy centers, and the additional cost of substitution to a single use duodenoscope may be even more significant for the centers with lower volume.

In addition, no manufacturers of single use duodenoscope 
can meet the demand of complete replacement of reusable duodenoscope as the manufacturing capability cannot simply keep up with the total number of ERCP procedure being performed. Thus, it is critical to identify the patient groups who may benefit the most with the use of single duodenoscope, such as immunocompromised patients and immuno-suppressed transplant patients. The experts, in collaboration with the manufacturers are currently preparing a position statement regarding its ethical and justifiable allocation.

Given the significant financial burden of single use duodenoscope, alternative methods, such as disposable cap, mounted at the tip of duodenoscope, is also being considered. The details of this modality are not within the scope of this review. Suffice to say however, that if proven equivalent to single use duodenoscope in terms of its infection prevention rate, this method may present a more economical alternative to single use duodenoscope. It is however unlikely to be superior to duodenoscope given the fact that the duodenoscope itself is being inserted to patients' gastrointestinal lumen and will be reused in others once the sterilization process takes place.

\section{CONCLUSIONS}

Considering the transmission of infectious microorganisms reported in recent years, the use of a disposable duodenoscope is not a matter of choice. However, there is a need for buffer measures to suppress excessive rise in treatment costs. It can be used by small volume institutions that do not want to invest in capital equipment and it is useful when using at operating room or emergency room in an emergency situation. In addition, it can be used to stent placement in patients with cholangiocarcinoma or in in-patients with increased risk of transmission of their own infection. ${ }^{16}$ The complete disposable duodenoscope released so far is a product of two companies. In the near future, the use of disposable duodenoscope may become more popular due to problems such as the spread of more dangerous infectious microorganisms, an increase in labor costs, price reduction due to mass production and the increased cost of maintaining facilities. However, prior to its generalized adoption to current practice, some adjustments are still needed. After the use for example, proper methods of its collection, safe disassembly and redistribution of its components for potential recycling must be mapped out. As it stands, healthcare institutions determined to use this technology is also subjected to the mercy of the supply and distribution channel (pre and post use) of the manufacturer. Although unclear, this may cause additional financial burden to the end-user, namely the healthcare institutions.

Lastly, given the current state of production capability and environmental consideration, the clinicians who would be at the forefront of its use must formulate and prioritize proper indication for the device. This will require creation of guidelines and recommendations from respective gastrointestinal endoscopic societies. At present, the population with compromised immunological functions such as those with organ transplantation, active malignancy and acquired immunocompromised state ought to be considered appropriate for its use. Further studies are required to delineate clinical benefits in the aforementioned populations.

\section{Conflicts of Interest}

Sunguk Jang is a paid consultant to Boston Scientific. The author has no potential conflicts of interest.

Funding

None.

Author Contributions

Conceptualization: Kihyun Ryu, Sunguk Jang

Data curation: KHR

Investigation: KHR, SUJ

Methodology: KHR

Visualization: KHR, SUJ

Writing-original draft: KHR, SUJ

ORCID

Kihyun Ryu

Sunguk Jang

https://orcid.org/0000-0003-0595-6776 https://orcid.org/0000-0001-9837-0322

\section{REFERENCES}

1. Ha J, Son BK. Current issues in duodenoscope-associated infections: now is the time to take action. Clin Endosc 2015;48:361-363.

2. Rutala WA, Weber DJ. Disinfection and sterilization in health care facilities: an overview and current issues. Infect Dis Clin North Am 2016;30:609-637.

3. Aumeran C, Poincloux L, Souweine B, et al. Multidrug-resistant klebsiella pneumoniae outbreak after endoscopic retrograde cholangiopancreatography. Endoscopy 2010;42:895-899.

4. Epstein L, Hunter JC, Arwady MA, et al. New Delhi metallo- $\beta$-lactamase-producing carbapenem-resistant escherichia coli associated with exposure to duodenoscopes. JAMA 2014;312:1447-1455.

5. Wendorf KA, Kay M, Baliga C, et al. Endoscopic retrograde cholangiopancreatography-associated AmpC escherichia coli outbreak. Infect Control Hosp Epidemiol 2015;36:634-642.

6. Kola A, Piening B, Pape U-F, et al. An outbreak of carbapenem-resistant OXA-48 - producing klebsiella pneumonia associated to duodenoscopy. Antimicrob Resist Infect Control 2015;4:8.

7. Muscarella LF. Risk of transmission of carbapenem-resistant enterobacteriaceae and related "superbugs" during gastrointestinal endoscopy. World J Gastrointest Endosc 2014;6:457-474.

8. Verfaillie CJ, Bruno MJ, Voor in 't Holt AF, et al. Withdrawal of a novel-design duodenoscope ends outbreak of a VIM-2-producing pseudomonas aeruginosa. Endoscopy 2015;47:493-502.

9. Loeve AJ. Investigational report on a TJF-Q180V duodenoscope following contamination after cleaning and disinfection. Available on request from the Dutch Healthcare Inspectorate/National Institute for Public 
Health and the Environment. Bilthoven, The Netherlands, 2012.

10. Ross AS, Baliga C, Verma P, Duchin J, Gluck M. A quarantine process for the resolution of duodenoscope-associated transmission of multidrug-resistant Escherichia coli. Gastrointest Endosc 2015;82:477-483.

11. Rauwers AW, Voor In 't Holt AF, Buijs JG, et al. High prevalence rate of digestive tract bacteria in duodenoscopes: a nationwide study. Gut 2018;67:1637-1645.

12. Rex DK, Sieber M, Lehman GA, et al. A double-reprocessing high-level disinfection protocol does not eliminate positive cultures from the elevators of duodenoscopes. Endoscopy 2018;50:588-596.

13. Vickery K, Ngo Q-D, Zou J, Cossart YE. The effect of multiple cycles of contamination, detergent washing, and disinfection on the development of biofilm in endoscope tubing. Am J Infect Control 2009;37:470-475.

14. Pajkos A, Vickery K, Cossart Y. Is biofilm accumulation on endoscope tubing a contributor to the failure of cleaning and decontamination? J Hosp Infect 2004;58:224-229.

15. Naryzhny I, Silas D, Chi K. Impact of ethylene oxide gas sterilization of duodenoscopes after a carbapenem-resistant Enterobacteriaceae outbreak. Gastrointest Endosc 2016;84:259-262.

16. Kim S, Russell D, Mohamadnejad M, et al. Risk factors associated with the transmission of carbapenem-resistant enterobacteriaceae via contaminated duodenoscopes. Gastrointest Endosc 2016;83:1121-1129.

17. Chan JK, Ng I, Ang JP, et al. Randomised controlled trial comparing the Ambu ${ }^{\circ}$ aScopeTM2 with a conventional fibreoptic bronchoscope in orotracheal intubation of anaesthetised adult patients. Anaesth Intensive Care 2015;43:479-484.
18. Mager R, Kurosch M, Höfner T, Frees S, Haferkamp A, Neisius A. Clinical outcomes and costs of reusable and single-use flexible ureterorenoscopes: a prospective cohort study. Urolithiasis 2018;46:587-593.

19. Terjesen CL, Kovaleva J, Ehlers L. Early assessment of the likely cost effectiveness of single-use flexible video bronchoscopes. Pharmacoecon Open 2017;1:133-141.

20. Hennessey DB, Fojecki GL, Papa NP, Lawrentschuk N, Bolton D. Single-use disposable digital flexible ureteroscopes: an ex vivo assessment and cost analysis. BJU Int 2018;121 (Suppl 3):55-61.

21. Muthusamy VR, Bruno MJ, Kozarek RA, et al. Clinical evaluation of a single-use duodenoscope for endoscopic retrograde cholangiopancreatography. Clin Gastroenterol Hepatol 2020;18:2108-2117.e3.

22. Cotton PB, Eisen G, Romagnuolo J, et al. Grading the complexity of endoscopic procedures: results of an ASGE working party. Gastrointest Endosc 2011;73:868-874.

23. Ross AS, Bruno MJ, Kozarek RA, et al. Novel single-use duodenoscope compared with 3 models of reusable duodenoscopes for ERCP: a randomized bench-model comparison. Gastrointest Endosc 2020;91:396403

24. Bang JY, Sutton B, Hawes R, Varadarajulu S. Concept of disposable duodenoscope: at what cost? Gut 2019;68:1915-1917.

25. Alrabaa SF, Nguyen P, Sanderson R, et al. Early identification and control of carbapenemase-producing klebsiella pneumoniae, originating from contaminated endoscopic equipment. Am J Infect Control 2013;41:562564. 